\title{
REFLEXIVITY OF PAIRS OF SHIFTS
}

\author{
MAREK PTAK
}

(Communicated by John B. Conway)

\begin{abstract}
In the present paper the reflexivity of a WOT-closed algebra generated by certain pairs of commuting shifts, which are not necessarily doubly commuting, is proved.
\end{abstract}

In what follows $B(\mathbf{H})$ denotes the algebra of all (linear bounded) operators on a separable complex Hilbert space $\mathbf{H}$. Throughout the paper we simply write a subspace for a closed subspace of $\mathbf{H}$ and an algebra (of operators) on $\mathbf{H}$ for a subalgebra of $B(\mathbf{H})$ with the unit (the identity on $\mathbf{H}$ ). If $\mathscr{S}$ is a subset of $B(\mathbf{H})$ then $\mathscr{U}(\mathscr{S})$ stands for the WOT (Weak Operator Topology)closed algebra generated by $\mathscr{S}$ while Lat $\mathscr{S}$ denotes the lattice of all invariant subspaces for all operators from $\mathscr{S}$. For operators $T_{1}, \ldots, T_{n}$ on $\mathbf{H}$ we write in the sequel $\mathscr{U}\left(T_{1}, \ldots, T_{n}\right)$, Lat $\left(T_{1}, \ldots, T_{n}\right)$, respectively, instead of $\mathscr{U}\left(\left\{T_{1}, \ldots, T_{n}\right\}\right)$ and $\operatorname{Lat}\left(\left\{T_{1}, \ldots, T_{n}\right\}\right)$. AlgLat $\mathscr{S}$ stands for the algebra of all operators on $\mathbf{H}$ which leave invariant all subspaces from Lat $\mathscr{S}$. An algebra $\mathscr{A}$ is called reflexive if and only if $\mathscr{A}=$ AlgLat $\mathscr{A}$. A family $\mathscr{S} \subset B(\mathbf{H})$ is called reflexive if the algebra $\mathscr{U}(\mathscr{S})$ is reflexive. An operator $T \in B(\mathbf{H})$ is called reflexive if $\mathscr{S}=\{T\}$.

Deddens [1] proved the reflexivity of an isometry using the Wold decomposition and a model for a shift. If a pair of commuting isometries is considered then a Wold-type decomposition need not exist [7]. Another difficulty is a lack of any model for a pair of commuting shifts in a general situation. In [3] the reflexivity of a pair $\left\{V_{1}, V_{2}\right\}$ of doubly commuting isometries (i.e. $V_{1}, V_{2}$ commute and so do $V_{1}, V_{2}^{*}$ ) was proved. Thus a pair of doubly commuting shifts is also reflexive. In this paper we prove, using a model from [3], the reflexivity of certain pairs of shifts, which are not necessarily doubly commuting.

Let $S$ denote a set of all pairs of nonnegative integers and $G$ be a set of all pairs of integers. Following [3], we introduce some notations and definitions. Namely, a subset $X \subset G$ is called a diagram if $\phi \in X, s \in S$ imply $\phi+s \in X$. The set of all diagrams is denoted by $\mathbf{X}$. For $\phi \in G$ we define $E_{\phi}=\{X \in \mathbf{X}$ : $\phi \in X\}$. It is obvious that $E_{\phi} \subset E_{\phi+s}(\phi \in G, s \in S)$. Let $\mathscr{B}$ be a $\sigma$-algebra

Received by the editors July $31,1989$.

1980 Mathematics Subject Classification (1985 Revision). Primary 47D25; Secondary 47B35.

Key words and phrases. Reflexivity, pairs of commuting shifts, modified unilateral translation. 
generated by all $E_{\phi}(\phi \in G)$ and $\mu$ be a positive finite measure on $(\mathbf{X}, \mathscr{B})$.

Let us denote $\mathscr{H}=\oplus_{\phi \in G} H_{\phi}$ where $H_{\phi}=\mathbf{H}$ and consider a space $\mathbf{K}$ of all measurable functions $f: \mathbf{X} \rightarrow \mathscr{H}$ such that $\int\|f(X)\|^{2} d \mu<\infty$ and $f(X) \in$ $\oplus_{\phi \in X} H_{\phi}$ (we identify functions equal $\mu$ a.e.). $\mathbf{K}$ is a Hilbert space with an inner product $(f, g)=\int(f(X), g(X))_{\not{Z}} d \mu(X)$. We note that each element of $\mathbf{K}$ can be written as follows: $f=\sum_{\phi \in G} f_{\phi}(\cdot) e_{\phi}$, where $f_{\phi}: \mathbf{X} \rightarrow \mathbf{H}, f_{\phi} \in$ $L^{2}(\mathbf{X}, \mathscr{B}, \mu, \mathbf{H})$ and $\sum_{\phi \in G}\left\|f_{\phi}\right\|^{2}<\infty ;$ also, $f_{\phi}(X)=0 \mu$ a.e. on $\mathbf{X}-E_{\phi}, e_{\phi}$ means that $f_{\phi}(X) e_{\phi}$ is an element of $H_{\phi}$ for $X \in \mathbf{X}$. Now, we can define operators $T_{s}$ on $\mathbf{K}, s \in S$. If $f=\sum_{\phi \in G} f_{\phi}(\cdot) e_{\phi}$, then

$$
T_{s} f=\sum_{\phi \in G} f_{\phi}(\cdot) e_{\phi+s} .
$$

Since $\mathbf{X}$ is the set of all diagrams, $T_{s} f \in \mathbf{K}$ and, therefore, $T_{s}$ are well-defined. Our main result is the following:

Theorem. $A$ WOT-closed algebra generated by $T_{s}, s \in S$ is reflexive.

We denote the above algebra by $\mathscr{A}$. Let us note that $\mathscr{A}=\mathscr{U}\left(T_{(1,0)}, T_{(0,1)}\right)$, thus, the Theorem implies that the pair $\left(T_{(1,0)}, T_{(0,1)}\right)$ is reflexive. Now we consider the following examples.

Example 1. If $\mu=\delta_{S}$ (the point mass at $S$ ), then the pair $\left(T_{(1,0)}, T_{(0,1)}\right)$ is a model for a pair of doubly commuting shifts [Theorem 1, 7]. The reflexivity of such pairs was proved in [4] and [5].

Example 2. [Example 1,7]. Let $X_{0}=\{(i, j): i \geq 0$ or $j \geq 0\}$. If $\mu=\delta_{X_{0}}$ then the pair $\left(T_{(1,0)}, T_{(0,1)}\right)$ is not doubly commuting. The Theorem implies that such a pair is reflexive.

Gaspar and Suciu proved [Theorem 6, 2] that each isometric semi-group is an orthogonal sum of a unitary semi-group, a left evanescent one, and a modified unilateral translation (for definitions, see [2]). A consequence of our theorem is the following:

Corollary 3. A modified unilateral translation on the semi-group of pairs of nonnegative integers is reflexive.

Proof. The $T_{s}, s \in S$, for a semi-group. If we consider the measure $\mu=\delta_{X_{0}}$ (as in Example 2), then $T_{s}, s \in S$, is a modified natural unilateral translation of the defect space $\mathbf{H}$ on the above semi-group $S$ ([2]). A modified unilateral translation is unitary equivalent to a modified natural unilateral translation; thus, it is reflexive.

Let us start proving our theorem. The following remarks and lemmas are needed.

If $\phi \in G$, then we denote $S_{\phi}=\phi+S$ and $L_{\phi}=\left\{f \in K: f(X) \in \oplus_{\psi \in S_{\phi}} H_{\psi}\right.$, $\mu$ a.e., $f(X)=0 \mu$ a.e. on $\left.\mathbf{X}-E_{\phi}\right\}$. 
Remark 4. $\mathbf{K}=\overline{\operatorname{span}\left\{L_{\phi}: \phi \in G\right\}}$

Proof. If $f \in \mathbf{K}$, then $f=\sum_{\phi \in G} f_{\phi}(\cdot) e_{\phi}$, where $f_{\phi}(X)=0 \mu$ a.e. on $\mathbf{X}-E_{\phi}$ and $f_{\phi}(X) e_{\phi} \in H_{\phi} \subset \oplus_{\psi \in S_{\phi}} \mathbf{H}_{\psi}$ for $X \in \mathbf{X}$; thus, $f_{\phi}(X) e_{\phi} \in L_{\phi}$. So the proof is finished.

Lemma 5. The subspace $L_{\phi}$ is invariant for $T_{s}$, if $s \in S, \phi \in G$.

Proof. Let $f \in L_{\phi}$ and $f=\sum_{\psi \in S_{\phi}} f_{\psi}(\cdot) e_{\psi}$ then, since $s \geq 0,\left(T_{s} f\right)(X)=$ $\sum_{\psi \in S_{\phi}} f_{\psi}(X) e_{\psi+s} \subset \oplus_{\psi \in S_{\phi}} \mathrm{H}_{\psi}$, for $X \in \mathbf{X}$. We know that $f(X)=0 \mu$ a.e. on $\mathbf{X}-E_{\phi}$, so, $f_{\phi}(X)=0 \mu$ a.e. on $\mathbf{X}-E_{\phi}$. Thus, $\left(T_{s} f\right)(X)=0 \mu$ a.e. on $\mathbf{X}-E_{\phi}$.

The subspace $L_{\phi}$ is unitary equivalent to a space $H^{2}\left(\Gamma^{2}\right) \otimes L^{2}\left(E_{\phi},\left.\mu\right|_{E_{\phi}}, \mathbf{H}\right)$, where $H^{2}\left(\Gamma^{2}\right)$ is the Hardy space ( $\Gamma$ denotes a unit circle). Let $U_{\phi}$ define this unitary equivalence, i.e.,

$$
\left(U_{\phi} \sum_{t \in S_{\phi}} f_{t}(\cdot) e_{t}\right)=\sum_{t \in S_{\phi}} z^{t-\phi} \otimes f_{t}(\cdot)
$$

We have also:

$$
\begin{aligned}
U_{\phi} T_{s} \sum_{t \in S_{\phi}} f_{t}(\cdot) e_{t} & =U_{\phi} \sum_{t \in S_{\phi}} f_{t}(\cdot) e_{s+t}=\sum_{t \in S_{\phi}} z^{s+t-\phi} \otimes f_{t}(\cdot) \\
& =\sum_{t \in S_{\phi}} M_{z^{s}} z^{t-\phi} \otimes f_{t}(\cdot)=\left(M_{z^{s}} \otimes I_{\phi}\right) \sum_{t \in S_{\phi}} z^{t-\phi} \otimes f_{t}(\cdot) \\
& =\left(M_{z^{s}} \otimes I_{\phi}\right) U_{\phi} \sum_{t \in S_{\phi}} f_{t}(\cdot) e_{t},
\end{aligned}
$$

where $M_{z^{s}}$ denotes a multiplication operator by $z^{s}=z_{1}^{s_{1}} z_{2}^{s_{2}}$ (here $z_{1}, z_{2}$ denote independent variables and $\left.s=\left(s_{1}, s_{2}\right)\right)$ in the space $H^{2}\left(\Gamma^{2}\right)$ and $I_{\phi}$ denotes identity operator in $L^{2}\left(E_{\phi},\left.\mu\right|_{E_{\phi}}, \mathbf{H}\right)$. Thus,

$$
U_{\phi} T_{s} U_{\phi}^{*}=M_{z^{s}} \otimes I_{\phi} \quad \text { for } \phi \in G, s \in S .
$$

Lemma 6. If $\phi \leq \psi, \phi, \psi \in G$, then $U_{\psi} T_{\psi-\phi} \mid L_{\phi}=U_{\phi}$.

Proof. Let $f \in L_{\phi}$ and $f=\sum_{t \in S_{\phi}} f_{t}(\cdot) e_{t}$ then

$$
\begin{aligned}
U_{\psi} T_{\psi-\phi} f & =U_{\psi} \sum_{t \in S_{\phi}} f_{t}(\cdot) e_{t+\psi-\phi}=\sum_{t \in S_{\phi}} z^{t+\psi-\phi-\psi} \otimes f_{t}(\cdot) \\
& =\sum_{t \in S_{\phi}} z^{t-\phi} \otimes f_{t}(\cdot)=U_{\phi} \sum_{t \in S_{\phi}} f_{t}(\cdot) e_{t}=U_{\phi} f .
\end{aligned}
$$

Lemma 7. If $\phi \leq \psi, \phi, \psi \in G$, then

$$
\left.U_{\phi} U_{\psi}^{*}\right|_{H^{2}\left(\Gamma^{2}\right) \otimes L^{2}\left(E_{\phi},\left.\mu\right|_{E_{\phi}}, \mathbf{H}\right)}=M_{z^{\psi-\phi}} \otimes I_{\phi} .
$$


Proof. Let $f=\sum_{t \in S} z^{t} \otimes f_{t}(\cdot)$, where $f_{t} \in L^{2}\left(E_{\phi},\left.\mu\right|_{E_{\phi}}, \mathbf{H}\right)$. Then

$$
\begin{aligned}
U_{\phi} U_{\psi}^{*} f & =U_{\phi} \sum_{t \in S} f_{t}(\cdot) e_{t+\psi}=\sum_{t \in S} z^{t+\psi-\phi} \otimes f_{t}(\cdot) \\
& =M_{z^{\psi-\phi}} \otimes I_{\phi} f .
\end{aligned}
$$

Lemma 8. If $\operatorname{Lat}\left\{T_{s}: s \in S\right\} \subset$ Lat $A$, then $A T_{s}=T_{s} A$ for $s \in S$.

Proof. Let $\phi \in G$. Then from Lemma 5, $L_{\phi} \in \operatorname{Lat}\left\{T_{s}: s \in S\right\} \subset \operatorname{Lat} A$, thus $\operatorname{Lat}\left\{\left.T_{s}\right|_{L_{\phi}}: s \in S\right\} \subset$ Lat $\left.A\right|_{L_{\phi}}$. Now, (2) implies Lat $\left\{M_{z^{s}} \otimes I_{\phi}: s \in S\right\}$ $=\operatorname{Lat}\left\{U_{\phi} T_{s} U_{\phi}^{*}: s \in S\right\} \subset$ Lat $\left.U_{\phi} A\right|_{L_{\phi}} U_{\phi}^{*}$. Since the algebra $\mathscr{U}\left(M_{z^{s}}: s \in S\right)$ is reflexive [Corollary 8.(1),4], [5], the algebra $\mathscr{U}\left(M_{z^{s}}: s \in S\right) \otimes \mathbb{C} I_{\phi}=\mathscr{U}\left(M_{z^{s}} \otimes\right.$ $I_{\phi}: s \in S$ ) is also reflexive [Theorem 9.18, Corollary 9.19,6]. Hence, $\left.U_{\phi} A\right|_{L_{\phi}} U_{\phi}^{*}$ $\in \mathscr{U}\left(M_{z^{s}} \otimes I_{\phi}: s \in S\right)$, so $\left.A\right|_{L_{\phi}} \in \mathscr{U}\left(\left.T_{s}\right|_{L_{\phi}}: s \in S\right)$, thus, $\left.\left.A\right|_{L_{\phi}} T_{S}\right|_{L_{\phi}}=\left.\left.T_{s}\right|_{L_{\phi}} A\right|_{L_{\phi}}$ and Remark 4 concludes the proof.

Now for $\phi \in G$ consider subspaces $M_{\phi}=\{f \in \mathbf{K}: f(X)=0$, $\mu$ a.e. on $\left.\mathbf{X}-E_{\phi}\right\}$. A sequence $\left\{\phi_{n}\right\} \subset G$ is called strongly increasing if and only if $\phi_{n}^{(i)}<\phi_{n+1}^{(i)}, i=1,2$ and $\phi_{k}^{(i)}$ are co-ordinates of $\phi_{k}$.

Lemma 9. If a sequence $\left\{\phi_{n}\right\} \subset G$ is strongly increasing then $M_{\phi_{n}} \subset M_{\phi_{k}}$ for $n<k$ and $\overline{\cup_{n \in \mathbb{N}} M_{\phi_{n}}}=\mathbf{K}$.

Proof. The first part of the assertion is an instantaneous consequence of the inclusion $E_{\phi_{k}} \subset E_{\phi_{n}}$. To prove the other part, let $f=\sum_{\psi \in G} f_{\psi}(\cdot) e_{\psi}$ and $f_{\psi}(X)=0$ for $X \notin E_{\psi}$. If $\psi \leq \phi_{n}$, then $E_{\psi} \subset E_{\phi_{n}}$. Thus, $\mathscr{X}-E_{\psi} \supset \mathbf{X}-E_{\phi_{n}}$; but, $f_{\psi}(X)=0$ for $X \in \mathscr{X}-E_{\psi}$; so, $f_{\psi}(X)=0$ for $X \in \mathbf{X}-E_{\phi_{n}}$. Hence, if $\psi \leq \phi_{n}$, then $f_{\psi}(\cdot) e_{\psi} \in M_{\phi_{n}}$. The sequence $\left\{\phi_{n}\right\}$ is strongly increasing, thus, for any $\psi \in G$ there is an $n$ such that $\psi \leq \phi_{n}$.

Proof of the theorem. Let $A \in B(\mathbf{K})$ and $\operatorname{Lat}\left\{T_{s}: s \in S\right\} \subset$ Lat $A$. Lemma 5 shows that $L_{\phi} \in \operatorname{Lat}\left\{T_{s}: s \in S\right\} \subset$ Lat $A$ for $\phi \in G$. Thus, as in Lemma 8, Lat $\left\{M_{z^{s}} \otimes I_{\phi}: s \in S\right\}=\operatorname{Lat}\left\{U_{\phi} T_{s} U_{\phi}^{*}: s \in S\right\} \subset$ Lat $\left.U_{\phi} A\right|_{L_{\phi}} U_{\phi}^{*}$. The algebra $\mathscr{U}\left(M_{z^{s}} \otimes I_{\phi}: s \in S\right)$ is reflexive [4,5], hence there is a sequence of operators $\hat{\sigma}_{n}^{\phi} \otimes I_{\phi}$ WOT-converging to $\left.U_{\phi} A\right|_{L_{\phi}} U_{\phi}^{*}$, where $\hat{\sigma}_{n}^{\phi}$ are polynomials of the multiplication operators $M_{z_{1}}, M_{z_{2}}$ in $H^{2}\left(\Gamma^{2}\right)$. Let us note that $\hat{\sigma}_{n}^{\phi}$ may be chosen jointly bounded (it will be needed in what follows). For example, $\hat{\sigma}_{n}^{\phi}$ may be chosen as in the proof of the Theorem in [5]. The bidisc fulfills assumptions of this Theorem, because it is starlike with $C^{2}$-boundary. According to that proof, an estimation is the following:

$$
\left\|\hat{\sigma}_{n}^{\phi}\right\| \leq\left\|\left.A\right|_{L_{\phi}}\right\|+1 \leq\|A\|+1 \stackrel{d f}{=} C .
$$

The above convergence shows also that $\left.U_{\phi} A\right|_{L_{\phi}} U_{\phi}^{*}=A_{\phi} \otimes I_{\phi}$, where $A_{\phi}$ is chosen adequately. 
Remark 4 implies that there is $\phi_{0}$ such that $L_{\phi_{0}} \neq\{0\}$ and we denote $\hat{\sigma}_{n}=\hat{\sigma}_{n}^{\phi_{0}}$. The sequence $\hat{\sigma}_{n} \otimes I_{\phi_{0}}$ WOT-converges to $\left.U_{\phi_{0}} A\right|_{L_{\phi_{0}}} ^{0} U_{\phi_{0}}^{*}=A_{\phi_{0}} \otimes I_{\phi_{0}}$, thus the sequence $U_{\phi_{0}}^{*}\left(\hat{\sigma}_{n} \otimes I_{\phi_{0}}\right) U_{\phi_{0}}$ WOT-converges to $\left.A\right|_{L_{\phi_{0}}}$. Let $\psi \geq \phi_{0}$. Then $T_{\psi-\phi_{0}} L_{\phi_{0}} \subset L_{\phi_{0}}$ (Lemma 5) and, in particular, $U_{\phi_{0}}^{*}\left(\hat{\sigma}_{n} \otimes I_{\phi_{0}}\right) U_{\phi_{0}}$ WOTconverges to $\left.A\right|_{L_{\phi_{0}}}$ on $T_{\psi-\phi_{0}} L_{\phi_{0}}$. Also, $T_{\psi-\phi_{0}} L_{\phi_{0}} \subset L_{\psi}$ (Lemma 6); thus, $U_{\psi} U_{\phi_{0}}^{*}\left(\hat{\sigma}_{n} \otimes I_{\phi_{0}}\right) U_{\phi_{0}} U_{\psi}^{*}$ WOT-converges to $\left.U_{\psi} A\right|_{L_{\psi}} U_{\psi}^{*}$ on $U_{\psi} T_{\psi-\phi_{0}} L_{\phi_{0}}$. By Lemma 6, $U_{\psi} T_{\psi-\phi_{0}} L_{\phi_{0}}=U_{\phi_{0}} L_{\phi_{0}}=H^{2}\left(\Gamma^{2}\right) \otimes L^{2}\left(E_{\phi_{0}},\left.\mu\right|_{E_{\phi_{0}}}, \mathbf{H}\right)$. Lemma $7 \mathrm{im}-$ plies that $U_{\phi_{0}} U_{\psi}^{*}=M_{z^{\psi-\phi_{0}}} \otimes I_{\phi_{0}}$ on $H^{2}\left(\Gamma^{2}\right) \otimes L^{2}\left(E_{\phi_{0}},\left.\mu\right|_{E_{\phi_{0}}}, H\right)$. Thus $U_{\phi_{0}} U_{\psi}^{*}$ commutes with $\hat{\sigma}_{n} \otimes I_{\phi_{0}}$. Hence, $\hat{\sigma}_{n} \otimes I_{\phi_{0}}$ WOT-converges to $\left.U_{\psi} A\right|_{L_{\psi}} U_{\psi}^{*}=$ $A_{\psi} \otimes I_{\psi}$ on $H^{2}\left(\Gamma^{2}\right) \otimes L^{2}\left(E_{\phi_{0}},\left.\mu\right|_{E_{\phi_{0}}}, \mathbf{H}\right)$. Therefore, $\hat{\sigma}_{n}$ WOT-converges to $A_{\psi}$ on $H^{2}\left(\Gamma^{2}\right)$ and so $\hat{\sigma}_{n} \otimes I_{\psi}$ WOT-converges to $A_{\psi} \otimes I_{\psi}=\left.U_{\psi} A\right|_{L_{\psi}} U_{\psi}^{*}$ on $H^{2}\left(\Gamma^{2}\right) \otimes L^{2}\left(E_{\psi},\left.\mu\right|_{E_{\psi}}, \mathbf{H}\right)$.

Let $\left\{\phi_{m}\right\} \subset G$ be a strongly increasing sequence with first element equal to $\phi_{0}$. For any $m$ let us define a sequence of operators

$$
\eta_{n}^{m}=U_{\phi_{m}}\left(\hat{\sigma}_{n} \otimes I_{\phi_{m}}\right) U_{\phi_{m}}^{*} .
$$

Hence, $\eta_{n}^{m}$ WOT-converges to $\left.A\right|_{L_{\phi_{m}}}(n \rightarrow \infty)$ and we also have $\left\|\eta_{n}^{m}\right\| \leq C$. Let us extend $\eta_{n}^{m}$ to the whole $M_{\phi_{m}}^{\phi_{m}}$ in the following way: we consider spaces $M_{\phi_{m}}^{l}=\left\{f \in M_{\phi_{m}}: f(X) \in \oplus_{\alpha \in S_{1}} H_{\alpha} \mu\right.$ a.e. $\}$ for all nonnegative integers $l$ and $\mathbf{l}=(l, l)$. It is easy to see that $M_{\phi_{m}}=\overline{\cup_{l} M_{\phi_{m}}^{l}}$. We define

$$
\eta_{n}^{m} f=T_{1+\phi_{m}}^{*} \eta_{n}^{m} T_{1+\phi_{m}} f
$$

$f \in M_{\phi_{m}}^{l}$. Let us note that $T_{1+\phi_{m}} f \in L_{\phi_{m}}$ for $f \in M_{\phi_{m}}^{l}$. Since $f(X) \in$ $\oplus_{\alpha \in S_{-1}} H_{\alpha} \mu$ a.e., thus $\left(T_{1+\phi_{m}} f(X) \stackrel{\oplus}{\in} \oplus_{\alpha \in S_{\phi_{m}}} H_{\alpha} \mu\right.$ a.e. We also have $f \in M_{\phi_{m}}$; thus, $f(X)=0 \mu$ a.e. on $\mathbf{X}-E_{\phi_{m}}$. If $(X)=\sum_{\alpha \in G} f_{\alpha}(X) e_{\alpha}$, then $f_{\alpha}(X)=0 \mu$ a.e. on $\mathbf{X}-E_{\phi_{m}}$ and

$$
\left(T_{1+\phi_{m}} f\right)(X)=\sum_{\alpha \in G} f_{\alpha}(X) e_{\alpha+1+\phi_{m}}=\sum_{\alpha \in G} f_{\alpha-1-\phi_{M}}(X) e_{\alpha}=0
$$

$\mu$ a.e. on $\mathbf{X}-E_{\phi_{m}}$. Hence $T_{1+\phi_{m}} f \in L_{\phi_{m}}$ and definition (4) is correct. Operators $\eta_{n}^{m}$ commute with $\left.T_{S}\right|_{L_{\phi_{m}}}$. Hence definition (4) gives well-defined extension (independent from (l)) of operators $\eta_{n}^{m}$ to $\cup_{l} M_{\phi_{m}}^{l}$. There is also $\left\|\eta_{n}^{m}\right\| \leq C$ on $L_{\phi_{m}}$; thus, from (4), $\left\|\eta_{n}^{m}\right\| \leq C$ on $M_{\phi_{m}}^{l}$ for all $l$. Now, we can extend $\eta_{n}^{m}$ to the whole $M_{\phi_{m}}$ with $\left\|\eta_{n}^{m}\right\| \leq C$. 
The following lemma is needed:

Lemma 10. If $m \leq k, n \in \mathbb{N}$, then $\eta_{n}^{m}=\left.\eta_{n}^{k}\right|_{M_{\phi_{m}}}$.

Proof. First we show that $\eta_{n}^{m} f=\eta_{n}^{k} f$ for $f \in L_{\phi_{m}}$.

$$
\eta_{n}^{k} f=T_{\phi_{k}-\phi_{m}}^{*} \eta_{n}^{k} T_{\phi_{k}-\phi_{m}} f=T_{\phi_{k}-\phi_{m}}^{*} U_{\phi_{k}}^{*}\left(\hat{\sigma}_{n} \otimes I_{\phi_{k}}\right) U_{\phi_{k}} T_{\phi_{k}-\phi_{m}} f,
$$

and by Lemma 6 we have

$$
\eta_{n}^{k} f=U_{\phi_{m}}^{*}\left(\hat{\sigma}_{n} \otimes I_{\phi_{k}}\right) U_{\phi_{m}} f=U_{\phi_{m}}^{*}\left(\hat{\sigma}_{n} \otimes I_{\phi_{m}}\right) U_{\phi_{m}} f=\eta_{n}^{m} f,
$$

since the range of $U_{\phi_{m}}$ is included in $H^{2}\left(\Gamma^{2}\right) \otimes L^{2}\left(E_{\phi_{m}},\left.\mu\right|_{E_{\phi_{m}}}, \mathbf{H}\right)$. (4) implies that $\eta_{n}^{m}=\eta_{n}^{k}$ on $M_{\phi_{m}}^{l}$ for all 1 ; thus, the equality is satisfied on the whole $M_{\phi_{m}}$, because $\eta_{n}^{m}, \eta_{n}^{k}$ are bounded.

Lemma 10 implies that we can define operators $\cup_{m} \eta_{n}^{m}$ on $\cup_{m} M_{\phi_{m}}$ and $\left\|\cup_{m} \eta_{n}^{m}\right\| \leq C$. Thus, there is sequence of operators $\beta_{n} \stackrel{d f}{=} \overline{\cup_{m} \eta_{n}^{m}}$ on $\mathbf{K}=$ $\overline{\cup_{m} M_{\phi_{m}}}$ (Lemma 9) and $\left\|\beta_{n}\right\| \leq C$. Operators $\eta_{n}^{m}$ on $L_{\phi_{m}}$ are polynomials in $\left.T_{(1,0)}\right|_{L_{\phi_{m}}},\left.T_{(0,1)}\right|_{L_{\phi_{m}}}$, so, by extention (4), $\eta_{n}^{m}$ on $M_{\phi_{m}}$ are also polynomials in $\left.T_{(1,0)}\right|_{M_{\phi_{m}}},\left.T_{(0,1)}\right|_{M_{\phi_{m}}}$. Hence, Lemma 6 and properties of the closure imply that $\beta_{n}$ are polynomials in $T_{(1,0)}, T_{(0,1)}$. Thus, to finish the proof we show that $\beta_{n}$ WOT-converges to $A$ on $\mathbf{K}$.

Let $f, g \in M_{\phi_{m}}^{1}$ for any $1, m$. Then commutativity of $A$ and $T_{s}, s \in S$, (Lemma 8) implies

$$
\begin{aligned}
\left(\beta_{n} f, g\right)-(A f, g) & =\left(\eta_{n}^{m} f, g\right)-(A f, g) \\
& =\left(T_{l+\phi_{m}}^{*} \eta_{n}^{m} T_{l+\phi_{m}} f, g\right)-\left(T_{1+\phi_{m}}^{*} A T_{l+\phi_{m}} f, g\right) \\
& =\left(T_{1+\phi_{m}}^{*}\left(\eta_{n}^{m}-A\right) T_{1+\phi_{m}} f, g\right) \\
& =\left(\left(\eta_{n}^{m}-A\right) T_{1+\phi_{m}} f, T_{1+\phi_{m}} g\right) .
\end{aligned}
$$

Since $T_{1+\phi_{m}} f, T_{1+\phi_{m}} g \in L_{\phi_{m}}$, and we have shown that $\eta_{n}^{m}$ WOT-converges to $A$ on $L_{\phi_{m}}$, then

$$
\left(\beta_{n} f, g\right)-(A f, g) \rightarrow 0 \text { as } n \rightarrow \infty .
$$

The operators $\beta_{n}$ are jointly bounded; hence, $\beta_{n}$ WOT-converges to $A$ on $M_{\phi_{m}}$ for all $m$ and also on $\mathbf{K}$ from Lemma 9.

Remark 11. The Theorem (with the same proof) holds true, when $S$ is the set of all finite sequences of non-negative integers.

\section{REFERENCES}

1. J. A. Deddens, Every isometry is reflexive, Proc. Amer. Math. Soc., 28 (1971), 509-511.

2. D. Gaspar and N. Suciu, On the structure of isometric semigroups, Operator Theory: Adv. Appl. 14, Birkhäuser Verlag Besel, 1984, 125-139. 
3. K. Horak and V. Müller, Functional model for commuting isometries, Czech. Math. J. 2 (1989), 370-379.

4. M. Ptak, On the reflexivity of pairs of isometries and of tensor products of some operator algebras, Stud. Math. 83 (1986), 47-55.

5. _ Reflexivity of multiplication operators in certain domains in $\mathbb{C}^{N}$, Bull. Acad. Polon. Sci. Ser. Math. 37 (1989).

6. H. Radjavi and P. Rosenthal, Invariant subspaces, Springer-Verlag, New York, Heidelberg, Berlin, 1973.

7. M. Slocinski, On the Wold-type decomposition of a pair of commuting isometries, Ann. Polon. Math. 37 (1980), 255-262.

Institute of Mathematics, University of AgRiculture, Ul18 StyczNia 6, 30-045, Kraków, Poland 